\title{
2. On an Elaboration of M. Kac's Theorem Concerning Eigenvalues of $-\Delta$ in a Region with Randomly Distributed Small Obstacles
}

\author{
By Shin OzawA \\ Department of Mathematics, University of Tokyo \\ (Communicated by Kôsaku YosidA, M. J. A., Jan. 12, 1983)
}

Let $\Omega$ be a bounded domain in $R^{3}$ with smooth boundary $\gamma$. Let $0 \leq \mu_{1}(\varepsilon ; w(m)) \leq \mu_{2}(\varepsilon ; w(m)) \leq \cdots$ be the eigenvalues of $-\Delta(=-\operatorname{div}$ grad) in $\Omega_{\varepsilon, w(m)}=\Omega \backslash \overline{\bigcup_{i=1}^{m} B\left(\varepsilon ; w_{i}^{(m)}\right)}$ under the Dirichlet condition on its boundary. We arrange them repeatedly according to their multiplicities. Here $B(\varepsilon ; w)=\left\{x \in \boldsymbol{R}^{3} ;|x-w|<\varepsilon\right\}$ and $w(m)$ denotes the set of $m$ points $\bigcup_{i=1}^{m}\left\{w_{i}^{(m)}\right\}$. Let $V(x)$ be $C^{1}$ function on $\bar{\Omega}$ satisfying $V(x) \geq 0$ and

$$
\int_{\Omega} V(x) d x=1
$$

We consider $\Omega$ as the probability space with probability density $V(x) d x$.

Kac's theorem is the following

Theorem (Kac [1], Rauch-Taylor [5]). Fix $k$ and $\alpha>0$. Then

$$
\lim _{m \rightarrow \infty} \boldsymbol{P}\left(w(m) \in \Omega^{m} ;\left|\mu_{k}(\alpha / m ; w(m))-\mu_{k}^{V}\right|<\varepsilon\right)=1
$$

for any $\varepsilon>0$. That is, $\mu_{k}(\alpha / m ; w(m))$ tends to $\mu_{k}^{V}$ in probability. Here $\mu_{k}^{V}$ is the $k$-th eigenvalue of $-\Delta+4 \pi \alpha V(x)$ in $\Omega$ under the Dirichlet condition on $\gamma$.

In this note we give an elaboration of Kac's theorem. We have the following

Theorem 1. Fix $k$ and $\alpha>0$. Then

$$
\lim _{m \rightarrow \infty} \boldsymbol{P}\left(w(m) \in \Omega^{m} ;\left|\mu_{k}(\alpha / m ; w(m))-\mu_{k}^{V}\right|<\varepsilon m^{-\beta}\right)=1
$$

for any $\varepsilon>0$ and any fixed $\beta \in[0,1 / 4)$.

Remark. Kac [1] proved his result by using the theory of Wiener sausage in case $V(x)=(\text { volume of } \Omega)^{-1}$. After Kac [1], Rauch-Taylor [5] gave the result for general $V(x)$ by combining functional analysis of operators and the Feynmann-Kac formula. See also Simon [6], Papanicolaou-Varadhan [4].

Our proof of Theorem 1 is quite different from [1], [5]. The main idea is to use perturbational calculus using Green's function of $-\Delta$. A direct construction of an approximate Green's function of $-\Delta$ in $\Omega_{\alpha / m, w(m)}$ under the Dirichlet condition on $\partial \Omega_{\alpha / m, w(m)}$ in terms of Green's function of $-\Delta$ in $\Omega$ under the Dirichlet condition on $\gamma$ enables us to give a remainder estimate in Theorem 1. For the method using 
Green's function the reader may refer to [3].

By the use of Green's function we get also the result on an asymptotic behaviour of $\mu_{k}(\alpha / m ; w(m))$ when $w(m)$ is dispersed in a specific configuration (for example, lattice configuration) as $m \rightarrow \infty$. Both proofs of probabilistic result (Theorem 1) and deterministic result proceed in parallel.

A sequence $w(m), m=1,2, \ldots$ is said to be of class $\mathscr{F}$ if the following conditions (F-1), (F-2), (F-3) for $w(m)$ are satisfied:

$$
w_{i}^{(m)} \in \Omega, \quad \min _{i \neq j}\left|w_{i}^{(m)}-w_{j}^{(m)}\right| \geq C_{0} m^{-1+\nu}
$$

for some constant $C_{0}$ independent of $m$. Here $\nu$ is a fixed constant satisfying $0 \leq \nu<1 / 3$.

(F-2) There exists a constant $C_{\xi}^{*}$ independent of $m$ (possibly depending on $\xi$ ) such that the following holds for any $\xi>0$.

$$
\max _{m} \frac{1}{m^{2}} \sum_{\substack{i, j=1 \\ i \neq j}}^{m}\left|w_{i}^{(m)}-w_{j}^{(m)}\right|^{-3+\xi} \leq C_{\xi}^{*}<\infty .
$$

(F-3) Let $f_{h}, h=1,2,3, \cdots$ be an arbitrary fixed family of continuous functions on $\bar{\Omega}$ satisfying

$$
\max _{x \in \Omega}\left|f_{h}(x)\right| \leq C^{-h}
$$

for some constant $C>1$. Fix an arbitrary $0 \leq \delta<1 / 2$ and $\lambda>0$. Then

$$
\lim _{m \rightarrow \infty} m^{\delta}\left(\sup _{h} C^{h / 2}\left(\frac{1}{m} \sum_{i=1}^{m} f_{h}\left(w_{i}^{(m)}\right)-f_{h}(x) V(x) d x\right)\right)=0
$$

and

$$
\begin{gathered}
\lim _{m \rightarrow \infty} m^{\delta}\left(\operatorname { s u p } _ { h } C ^ { h / 2 } \left(\frac { 1 } { m } \sum _ { i _ { 1 } = 1 } ^ { m } \left\{\frac{1}{m} \sum_{\substack{i_{2} \neq i_{1} \\
i_{2}=1}}^{m} G_{(\lambda)}\left(w_{i_{1}}^{(m)}, w_{i_{2}}^{(m)}\right) f_{h}\left(w_{i_{2}}^{(m)}\right)\right.\right.\right. \\
\left.\left.\left.-\left(\boldsymbol{G}_{(\lambda)} V f_{h}\right)\left(w_{i_{1}}^{(m)}\right)\right\}^{2}\right)\right)=0
\end{gathered}
$$

where $G_{(\lambda)}(x, y)$ denotes Green's function of $-\Delta+\lambda(\lambda \geq 0)$ in $\Omega$ under the Dirichlet condition on $\gamma$ and $G(\lambda)$ denotes the integral operator given by

We have the following

$$
(G(\lambda) f)(x)=\int_{\Omega} G_{(\lambda)}(x, y) f(y) d y .
$$

Proposition 1. Fix $k$ and $\alpha>0$. Assume that $\{w(m)\}_{m=1}^{\infty} \in \mathscr{F}$. Then

$$
\lim _{m \rightarrow \infty}\left|\mu_{k}(\alpha / m ; w(m))-\mu_{k}^{V}\right|=0\left(m^{-\delta / 2}\right)
$$

holds for $\delta \in[0,1 / 2)$.

We see that

for any $\tilde{C}_{0} \in R$,

$$
\lim _{m \rightarrow \infty} \boldsymbol{P}\left(w(m) \in \Omega^{m} ; \min _{i \neq j}\left|w_{i}^{(m)}-w_{j}^{(m)}\right| \leq \tilde{C}_{0} m^{-1+\nu}\right)=0
$$

$$
\lim _{m \rightarrow \infty} \boldsymbol{P}\left(w(m) \in \Omega^{m} ;(\mathrm{F}-2) \text { does not hold }\right)=0
$$

and that the convergence in (1), (2) is the convergence in probability. 
These facts are used and essential in the proof of Theorem 1.

The details of the proof of Theorem 1 and related results will be given in [2]. The author here expresses his cordial gratitude to Prof. G. C. Papanicolaou for valuable discussions and encouragement.

\section{References}

[1] M. Kac: Probabilistic methods in some problem of scattering theory. Rocky Mountain J. of Math., 4, 511-538 (1974).

[2] S. Ozawa: On an elaboration of M. Kac's theorem of $-\Delta$ in a region with randomly distributed small obstacles (preprint).

[ 3 ] — : Eigenvalues of the Laplacian on wildly perturbed domain. Proc. Japan Acad., 58A, 419-421 (1982).

[4] G. C. Papanicolaou and S. R. S. Varadhan: Diffusion in region with many small holes. Lecture Notes in Control and Information, 75, 190-206 (1980).

[5] J. Rauch and M. Taylor: Potential and scattering theory on wildly perturbed domains. J. Funct. Analysis, 18, 27-59 (1975).

[6] B. Simon: Functional Integration and Quantum Physics. Academic Press (1979). 